\title{
Clustered inter-phrase or word context-dependent models for continuously read Japanese
}

\author{
Kazuhiro Kondo,*,** Yu-Hung Kao,** and Barbara Wheatley** \\ * Texas Instruments Tsukuba Research and Development Center Ltd., \\ 17, Miyukigaoka, Tsukuba, 305 Japan \\ ** Systems and Information Science Laboratory, Texas Instruments Inc., \\ 13510, North Central Expressway, MS238, Dallas, Texas 75243, USA
}

(Received 8 December 1994)

\begin{abstract}
This paper investigates methods to model inter-phrase or word context for continuous Japanese speech recognition. It is well known that in continuous speech, coarticulation between words or phrases induces allophonic variation of the beginning and ending phones in words or phrases. It was found that by compiling a network of contextdependent phonetic models which models these inter-word or inter-phrase context, recognition error reduction by $32 \%$ can be achieved compared to models which do not account for inter-word context with task-dependent training, i.e. models that were trained with the same vocabulary as the test set. A more dramatic error reduction of up to $43 \%$ was possible with task-independent training. However, this will significantly increase the number of phonetic models required to model the vocabulary. With digit models, the increase in the number of models is 4 to 5 fold. To overcome this increase, we clustered the inter-word/phrase context into a few phonetic classes. Using one class for consonant inter-word context and two classes for vowel context, the recognition accuracy on digit string recognition was found to be virtually equal to the accuracy with unclustered models, while the number of phonetic models required was reduced by more than $50 \%$.
\end{abstract}

Keywords: Word/phrase context, Context clustering, Continuous speech, Phonetic modeling, Speech recognition

PACS number: 43. 72. Ne, 43. 70. Hs

\section{INTRODUCTION}

In recent years, Hidden Markov Model (HMM) based speech recognition systems using contextdependent phoneme models, such as triphones, have demonstrated high recognition accuracy by taking into account the neighboring phonetic contexts in a word or phrase which strongly affect the realization of a phoneme. It is also a well known fact that in continuous speech, the same effect is seen at the boundary between adjacent words or phrases. It has been shown previously that modeling these interword coarticulation in continuous speech dramatically improves the recognition accuracy. ${ }^{1-4}$ ) How- ever, by introducing additional phoneme variation models to account for these coarticulations, the required number of models increases significantly. This will mean more memory is needed to store these models, and more computation will be needed to match additional context with input speech. In cases where the grammar does not significantly constrain the possible number of words or phrases in the search paths, the above increase will be prohibitive. To give an extreme example, with digit strings, where any digit can follow each other, the number of phoneme models increase from 96 for the inter-word context-independent models, to 461 when interword context is accounted for. The increase is 
nearly five fold. This is indeed an extreme case. However, even with a more generic recognition task, the increase in the number of models can be significant. For instance, Hwang et al. ${ }^{1)}$ report that with the 997-word DARPA Resource Management (RM) Task, the number of triphone models increase from 2,381 to 7,549, a three fold increase. Accordingly, efficient parsers which will reduce the added computation have been proposed previously. ${ }^{5,6}$ ) However, these parsers will have the added burden of judging and retaining the neighboring context including the optional pauses between words and phrases. Unlike these approaches, we propose to cluster the inter-word or phrase context into an extremely small number of clusters. We present results for the Japanese digit recognition task which prove that this clustering will have virtually no effect on the recognition accuracy. The required number of models with the proposed scheme is reduced by $50 \%$ compared to the unclustered case. Results using both task-dependent training and task-independent training will be given.

This paper is organized as follows. Section 2 gives a brief description of Japanese phontactic rules, Section 3 describes the conditions of our experiments, Section 4 describes the inter-word or phrase context modeling techniques proposed including the clustering schemes of these contexts. Finally, conclusions are given in Section 5.

\section{A BRIEF DESCRIPTION OF JAPANESE PHONOTACTICS}

Before going into the details on inter-word/phrase context, a brief description of Japanese phonotactic constraints will be given in this section. Figure 1 illustrates the Japanese phonotactic constraints.

In general, Japanese phonotactics is simple and very regular. A Japanese phrase or word is constructed of morae. A mora is either a (1) vowel, (2) a combination of a consonant and a vowel, (3) a consonant, the glide $/ y /$ and a vowel, or (4) the syllabic nasal /N/. The symbol /q/ can be also regarded as a mora, but actually stands for a geminate cluster. As described in footnote 1 in Fig. 1 , this symbols can be transcribed as an exceptional mora with one consonant in isolation.

From these rules, it can be observed that a word or phrase can be followed by any phone except $/ \mathrm{N} /$ and $/ \mathrm{q} /$, but can only be preceded by either a vowel or the phone $/ \mathrm{N} /$. This means that a word or

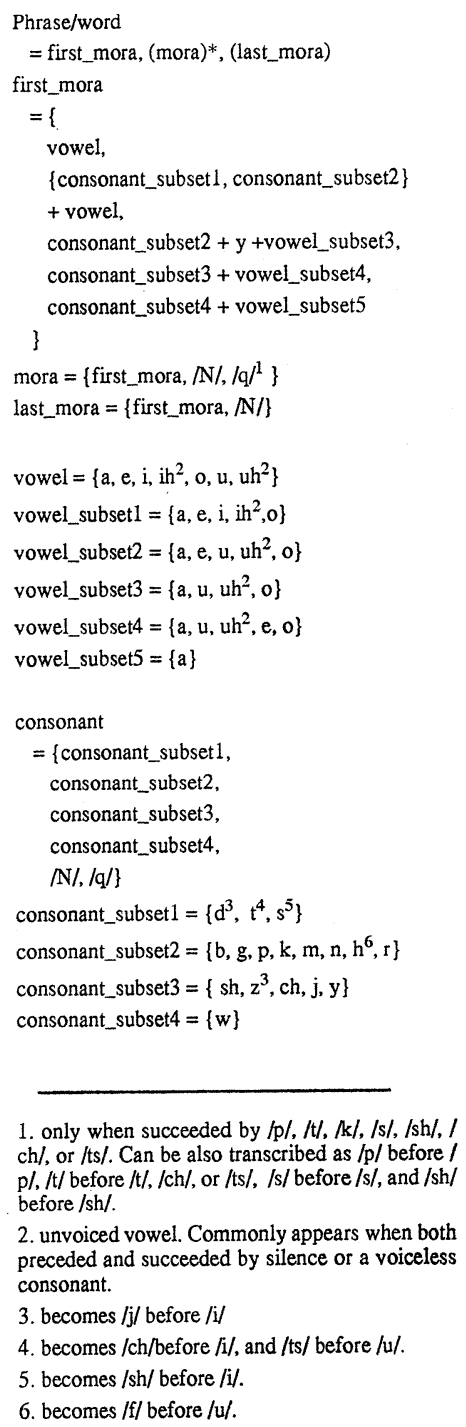

1. only when succeeded by $/ \mathrm{p} /, / \mathrm{t} /, \mathrm{k} /, / \mathrm{s} /, / \mathrm{sh} /, /$ $\mathrm{ch} /$, or $/ \mathrm{ts} /$. Can be also transcribed as /p/ before / $\mathrm{p} /, / \mathrm{t} /$ before $/ \mathrm{t} /, / \mathrm{ch} /$, or $/ \mathrm{ts} /, / \mathrm{s} / \mathrm{before} / \mathrm{s} /$, and $/ \mathrm{sh} /$ $\mathrm{p} /, / \mathrm{t} / \mathrm{befor} / \mathrm{t} / \mathrm{sef} / \mathrm{sh} /$.

2. unvoiced vowel. Commonly appears when both preceded and succeeded by silence or a voiceless consonant.

3. becomes /j/ before /i/

4. becomes $/ \mathrm{ch} /$ before $/ \mathrm{i} /$, and $/ \mathrm{ts} /$ before $/ \mathrm{u} /$.

5 . becomes $/ \mathrm{sh} /$ before $/ \mathrm{i} /$.

6. becomes /f/ before / $\mathrm{u}$.

Fig. 1 Japanese phonotactics.

phrase can have 25 different phonetic context on its right, but only 7 on its left, both including silences between words or phrases. Compared to other languages such as English, for which the phonotactic constraint does not significantly restrict the number of inter-word or phrase context, this is a much smaller number of contexts which must be accounted for. However, the number of context-sensitive phoneme models which must be added to model inter-word context is still quite prohibitive. In the following sections, clustering schemes which will 


\section{K. KONDO et al.: CLUSTERED INTER-PHRASE/WORD CONTEXT-DEPENDENT MODELS}

help cut down the required number of models will be investigated.

\section{EXPERIMENTAL CONDITIONS}

In this section, the experimental conditions for all tests will be described. The corpus used for both training and testing, as well as the baseline speech recognition system used in these experiments will be described.

\subsection{The Corpora}

Two corpora were used in these experiments. The first is a proprietary corpus collected for the voice dialing task. ${ }^{7)}$ The data in this corpus was used for task-dependent training, and all testing. The samples were recorded with a Digital Audio Tape (DAT) recorder, and later resampled at $8 \mathrm{kHz}$, in 16 bit linear format. The recordings were made with the same table-mounted electret condenser microphone in a relatively quiet environment. The collected speech consisted of command phrases, such as "uchi e denwa (call home)," as well as continuous digit strings. Only the latter was used for the tests described here. There were 221 speakers, 112 male and 109 female. Each speaker spoke 100 sentences; half were digit strings. Approximately $80 \%$ of the data for each sex was used for the fullset task-dependent training. We have also used limited data out of this training set in order to balance the amount of training data with the taskindependent training, which will be explained in the next paragraph. The remaining data was used for testing. No overlap in speakers between the test set and training set exists. Overall, a total of 5,447 male utterances and 5,380 female utterances were used for the full-set training. Among these, 867 male and 857 female utterances were used for the limited-set training, and 2,068 male and female utterances combined were used for all testing.

We used another corpus to investigate the performance of inter-word context-dependent models with task-independent training. The corpus used was the continuously read ATR 503 phonetically balanced sentences provided by the ASJ. ${ }^{8)}$ In this corpus, 503 phonetically balanced sentences, defined by ATR, were read by 64 participants from 15 institutions, 30 male and 34 female. The sampling rate was consistent at $16 \mathrm{kHz}$, but the other conditions including quantization bits, anti-alias filters, and microphones differ slightly by institutions. Most data were recorded in a virtually noiseless environment. A total of 3,317 female utterances, and 3,518 male utterances were used for the task-independent training.

\subsection{The Baseline Automatic Speech Recognition System}

All speech data used in the speech recognizer is sampled at $8 \mathrm{kHz}$. A tenth-order LPC analysis with a $20 \mathrm{~ms}$ frame duration, $30 \mathrm{~ms}$ Hamming window is applied. The LPC parameters are converted into speech feature vectors consisting of spectral energy vectors output from a filter bank consisting of $14 \mathrm{Mel}$-spaced filters, the short-term differences of these spectral energy measures, the speech level, and some voicing indicators. The total number of elements in this vector is 34 . A principal component based analysis designed to decorrelate its elements is applied to this vector. ${ }^{9)}$ Out of the transformed speech feature vector, 16 most significant elements are selected.

Continuous density HMM with a single multivariate Gaussian distribution per state is used to model these speech feature vectors along with a Viterbi-style maximum likelihood scoring. Each state in the model is associated with an input speech feature vector. The acoustics of each model state are defined by a Gaussian distribution mean vector which best matches speech feature vectors observed for this state during training, along with a single full covariance matrix pooled across all states in all models. The topology of the models used in this paper are all finite duration models without selfloops as shown in Fig. 2. This example is for a model that has feature vectors corresponding to three frames of speech. In this figure, $\mathrm{O}_{i}$ stands for

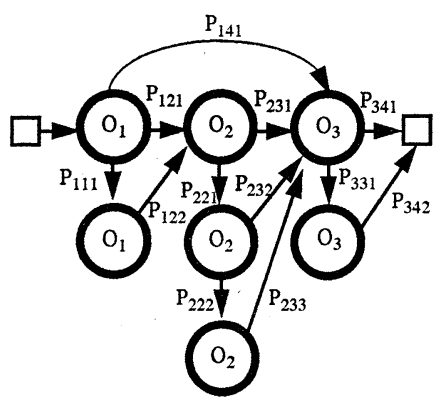

Fig. 2 Topology of HMM models used in all experiments. 
the state corresponding to the $i$-th feature vector, and $P_{i j k}$ stands for transition probability from the $i$-th to the $j$-th state after $k$ occurrences of state $i$. The number of different states for each model is proportional to the average duration of each phone. All states shown aligned in the vertical direction with the same indices are tied. Thus, there is an explicit limit in the maximum number of frames aligned with each state in each model. In most cases, we set this maximum limit to 3 frames per state. The model allows single skips between states. We have found consistently better performance with this configuration without loops, especially when the data tends to be noisy, and thus will be using this topology throughout our experiments. The only exception is the silence model which consists of a single state with a self loop.

\section{MODELING INTER-WORD/PHRASE CONTEXT DEPENDENCIES}

\subsection{Inter-Word/Phrase Context-Dependency} Modeling Procedures

In the automatic speech recognizer which will be used throughout this paper, word or phrase models are constructed from context-dependent phonetic models. We have used triphones as context-dependent units in our work described here. Using triphone models, inter-word or phrase context can be modeled easily by allowing multiple triphone at the beginning and end of a word. This is shown in Fig. 3 for the example of digit recognition. As shown in the figure, the grammar allows transition to any of the triphones which have, as its left context, the last phone in any word which can precede the current word. Silence context to account for pauses between words is also included. With digit strings, the number of triphones at the beginning is six. These model as its left context silence, $/ \mathrm{i} /, / \mathrm{N} /$, $/ \mathrm{o} /, / \mathrm{u} /$, and $/ \mathrm{a} /$. As stated in Section 2, the absolute maximum number of possible left context according to the Japanese phonotactic constraints is seven; silence, $/ \mathrm{a} /, / \mathrm{e} /, / \mathrm{i} /, / \mathrm{o} /, / \mathrm{u} /$, and $/ \mathrm{N} /$. Note that this excludes the two unvoiced vowels /ih/, and /uh/ which we have discriminated in our phone set, but have not distinguished with /i/ and /u/ whẹn modeling inter-word context.

Likewise, as its right context, the grammar allows 11 context; silence, $/ \mathrm{i} /, / \mathrm{n} /, / \mathrm{s} /, / \mathrm{y} /, / \mathrm{g} /, / \mathrm{r} /, / \mathrm{h} /, / \mathrm{k} /$, $\mid \mathrm{z} /$, and $/ \mathrm{m} /$. The Japanese phonotactic constraint allows a maximum of 23 ; silence, the five vowels

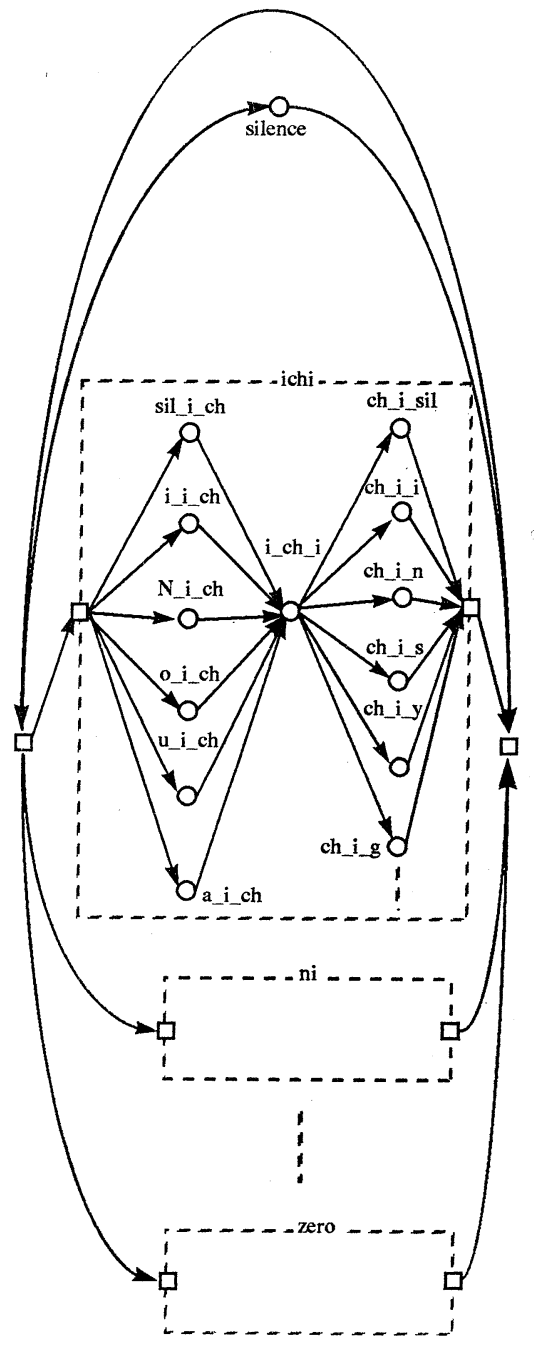

Fig. 3 An example recognition grammar for digit strings with inter-word context.

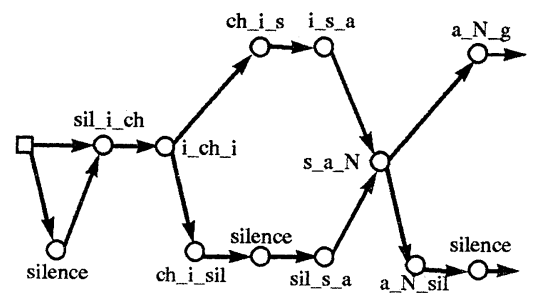

Fig. 4 An example training grammar for digit strings with inter-word context. 


\section{K. KONDO et al.: CLUSTERED INTER-PHRASE/WORD CONTEXT-DEPENDENT MODELS}

Table 1 Recognition error rates for inter-word context-dependent and independent models (task-dependent).

\begin{tabular}{|c|c|c|c|c|c|c|c|}
\hline \multirow{2}{*}{$\begin{array}{l}\text { Inter-word } \\
\text { context }\end{array}$} & \multirow{2}{*}{$\begin{array}{l}\text { Number of } \\
\text { models }\end{array}$} & \multicolumn{5}{|c|}{ Error rate } & \multirow{2}{*}{$\begin{array}{l}\text { Score } \\
\text { buffers }^{1}\end{array}$} \\
\hline & & Substitution & Deletion & Insertion & Word & Sentence & \\
\hline Dependent & 96 & 1.6 & 0.4 & 0.4 & 2.5 & 18.1 & 356 \\
\hline Independent & 461 & 1.0 & 0.4 & 0.3 & 1.7 & 13.3 & 1208 \\
\hline
\end{tabular}

1 Average number of score buffer slots needed per input speech frame.

$/ \mathrm{a} /, / \mathrm{e} /, / \mathrm{i} /, / \mathrm{o} /$, and $/ \mathrm{u} /$, and consonants $/ \mathrm{y} /, / \mathrm{d} /, / \mathrm{t} /$, $/ \mathrm{s} /, / \mathrm{b} /, / \mathrm{g} /, / \mathrm{p} /, / \mathrm{k} /, / \mathrm{m} /, / \mathrm{n} /, / \mathrm{h} /, / \mathrm{r} /, / \mathrm{sh} /, / \mathrm{z} /, / \mathrm{ch} /$, $\mathrm{j} /$, and $/ \mathrm{w} /$. Again, the unvoiced vowels, along with minor variations to consonants, namely /f/ and $/ \mathrm{ts} /$ have not been included.

Thus, the digit string example is one of the extreme cases where a large variation of inter-word context is seen since any digit can follow each other. We will be dealing with this task to illustrate the "upper bound" in performance improvements achievable with inter-word context-dependent models throughout this paper.

The selection of the appropriate inter-word context out of the possible paths was left completely unsupervised. The context can be restricted according to the neighboring word in the search path, thereby reducing the search space, at a cost of added complexity. However, we found empirically that leaving this context modeling path unsupervised does not have significant impact on the performance, and thus we decided to leave the search unrestricted.

The training of these context-dependent models can be accomplished using supervision grammars shown in Fig. 4. Each word model explicitly models the inter-word context, but also allows a secondary path which allows an optional silence between words, and accordingly models silence as context in this path.

Table 1 shows the recognition performance for models with both an inter-word context-dependent and independent models. The training was taskdependent in these experiments. In other words, the same corpus with the same vocabulary was used for training and testing, but the speakers and utterances were mutually exclusive, i.e. no speaker existed in both the training and test set, and each utterance was unique.

By introduction of inter-word context the word error rate decreases by $0.8 \%$, a relative decrease of $32 \%$. Interestingly, only substitution errors de- crease, not insertion or deletions. This seems to imply that the addition of interword context mainly enhances the discrimination between words. However, this behavior is highly correlated with the finite duration of the model topology used here. The number of triphone models required for the interword context-dependent models increases by almost five-fold compared to inter-word context-independent models, from 96 to 461 .

\subsection{Reduction of Inter-Word/Phrase Context through Clustering}

As we have seen in the previous sections, the number of inter-word or phrase context in Japanese is small by comparison to other languages such as English, but still is prohibitively large for practical tasks such as digit string recognition. Therefore, we investigated the possibility of reducing these context by clustering. We mainly used phonological classification to guide us in our clustering. All wordinternal context was modeled using conventional triphone models, as has been done in the previous experiments.

Table 2 summarizes the tested clustering schemes, while Fig. 5 through Fig. 12 illustrate an example word model for the word "ichi" which stands for "one" in Japanese. In scheme I, we clustered the consonants on the right side according to the place of articulation, while in scheme II, the consonants on right were clustered by manner of articulation. In scheme III, we further clustered all consonants on right into one. Scheme IV clusters all left vowels into front and back, while scheme $\mathrm{V}$ classifies all left vowels into high and mid or low vowels. All right context in schemes IV and V are treated in the same manner as scheme III. Scheme VI is an extreme case where all consonants and all vowels on either side are clustered into one each. In scheme VII, all phones are clustered into one on each side.

Table 3 summarizes the recognition error rates 
Table 2 Tested context clustering schemes.

\begin{tabular}{|c|c|c|c|}
\hline Clustering scheme & Context position & Cluster & Member phones \\
\hline \multirow{2}{*}{ None } & right & - & all 11 context modelled separately \\
\hline & left & - & all 6 context modelled separately \\
\hline \multirow[t]{2}{*}{ I } & right & $\begin{array}{l}\text { silence } \\
\text { vowel } \\
\text { bilabial } \\
\text { dental-alveolar } \\
\text { palatal-velar }\end{array}$ & $\begin{array}{l}\text { silence } \\
/ \mathrm{i} / \\
/ \mathrm{h} /, / \mathrm{m} / \\
/ \mathrm{s} /, / \mathrm{n} /, / \mathrm{z} /, / \mathrm{r} / \\
/ \mathrm{g} /, / \mathrm{y} /, / \mathrm{k} /\end{array}$ \\
\hline & left & - & all 6 context modelled separately \\
\hline \multirow[t]{2}{*}{ II } & right & $\begin{array}{l}\text { silence } \\
\text { vowel } \\
\text { nasals } \\
\text { stops } \\
\text { fricatives } \\
\text { liquid } \\
\text { glide }\end{array}$ & $\begin{array}{l}\text { silence } \\
/ \mathrm{i} / \\
/ \mathrm{n} /, / \mathrm{m} / \\
/ \mathrm{g} /, / \mathrm{k} / \\
/ \mathrm{s} /, / \mathrm{z} /, / \mathrm{h} / \\
/ \mathrm{r} / \\
/ \mathrm{y} /\end{array}$ \\
\hline & left & - & all 6 context modelled separately \\
\hline \multirow[t]{2}{*}{ III } & right & $\begin{array}{l}\text { silence } \\
\text { vowel } \\
\text { consonant }\end{array}$ & $\begin{array}{l}\text { silence } \\
\text { /i/ } \\
/ \mathrm{h} /, / \mathrm{m} /, / \mathrm{s} /, / \mathrm{n} /, / \mathrm{z} /, / \mathrm{r} /, / \mathrm{g} /, / \mathrm{y} /, / \mathrm{k} /\end{array}$ \\
\hline & left & - & all 6 context modelled separately \\
\hline \multirow[b]{2}{*}{ IV } & right & $\begin{array}{l}\text { silence } \\
\text { vowel } \\
\text { consonant }\end{array}$ & $\begin{array}{l}\text { silence } \\
/ \mathrm{i} / \\
/ \mathrm{h} /, / \mathrm{m} /, / \mathrm{s} /, / \mathrm{n} /, / \mathrm{z} /, / \mathrm{r} /, / \mathrm{g} /, / \mathrm{y} /, / \mathrm{k} /\end{array}$ \\
\hline & left & $\begin{array}{l}\text { silence } \\
\text { front vowels } \\
\text { back vowels } \\
\text { nasal }\end{array}$ & $\begin{array}{l}\text { silence } \\
/ \mathrm{i} / \\
/ \mathrm{a} /, / \mathrm{o} /, / \mathrm{u} / \\
/ \mathrm{N} /\end{array}$ \\
\hline \multirow{2}{*}{ V } & right & $\begin{array}{l}\text { silence } \\
\text { vowel } \\
\text { consonant }\end{array}$ & $\begin{array}{l}\text { silence } \\
/ \mathrm{i} / \\
/ \mathrm{h} /, / \mathrm{m} /, / \mathrm{s} /, / \mathrm{n} /, / \mathrm{z} /, / \mathrm{r} /, / \mathrm{g} /, / \mathrm{y} /, / \mathrm{k} /\end{array}$ \\
\hline & left & $\begin{array}{l}\text { silence } \\
\text { high vowel } \\
\text { mid-low vowel } \\
\text { nasal }\end{array}$ & $\begin{array}{l}\text { silence } \\
/ \mathrm{i} /, / \mathrm{u} / \\
/ \mathrm{a} /, / \mathrm{o} / \\
/ \mathrm{N} /\end{array}$ \\
\hline \multirow{2}{*}{ VI } & right & $\begin{array}{l}\text { silence } \\
\text { consonants } \\
\text { vowel }\end{array}$ & $\begin{array}{l}\text { silence } \\
/ \mathrm{n} /, / \mathrm{s} /, / \mathrm{y} /, / \mathrm{g} /, / \mathrm{r} /, / \mathrm{h} /, / \mathrm{k} /, / \mathrm{z} /, / \mathrm{m} / \\
/ \mathrm{i} /\end{array}$ \\
\hline & left & $\begin{array}{l}\text { silence } \\
\text { consonant } \\
\text { vowels }\end{array}$ & $\begin{array}{l}\text { silence } \\
/ \mathrm{N} / \\
/ \mathrm{i} /, / \mathrm{o} /, / \mathrm{u} /, / \mathrm{a} /\end{array}$ \\
\hline \multirow{2}{*}{ VII } & right & $\begin{array}{l}\text { silence } \\
\text { all phones }\end{array}$ & $\begin{array}{l}\text { silence } \\
/ \mathrm{i} /, / \mathrm{n} /, / \mathrm{s} /, / \mathrm{y} /, / \mathrm{g} /, / \mathrm{r} /, / \mathrm{h} /, / \mathrm{k} /, / \mathrm{z} /, / \mathrm{m} /\end{array}$ \\
\hline & left & $\begin{array}{l}\text { silence } \\
\text { all phones }\end{array}$ & $\begin{array}{l}\text { silence } \\
/ \mathrm{i} /, / \mathrm{N} /, / \mathrm{o} /, / \mathrm{u} /, / \mathrm{a} /\end{array}$ \\
\hline
\end{tabular}




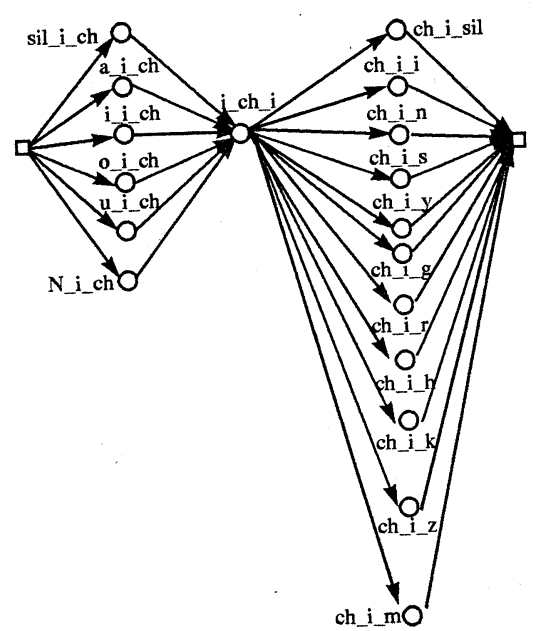

Fig. 5 Inter-word context-dependent word grammar for "ichi" (one in Japanese) without clustering.

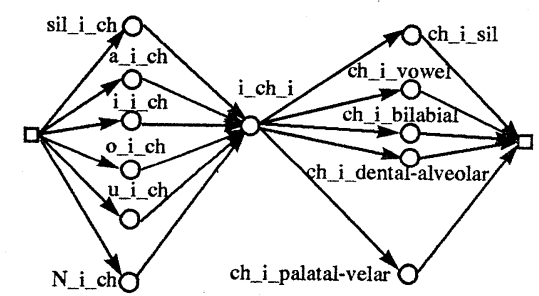

Fig. 6 Inter-word context-dependent word grammar for "ichi" (one in Japanese) clustered with scheme I.

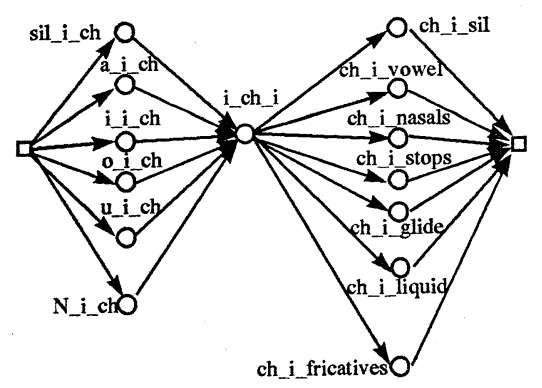

Fig. 7 Inter-word context-dependent word grammar for "ichi" (one in Japanese) clustered with scheme II.

for each scheme. Again, all training and testing were done on the digit string corpus. Surprisingly, the difference in performance between clustering

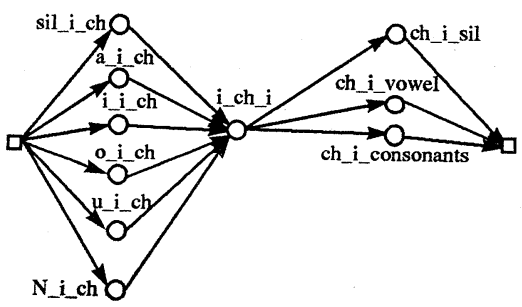

Fig. 8 Inter-word context-dependent word grammar for "ichi" (one in Japanese) clustered with scheme III.

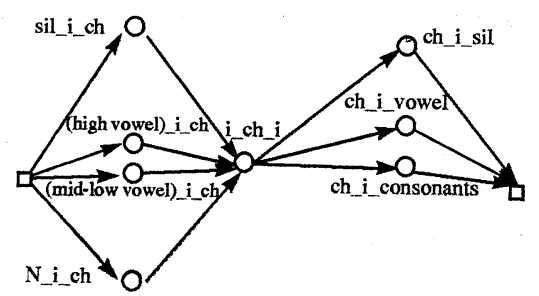

Fig. 9 Inter-word context-dependent word grammar for "ichi" (one in Japanese) clustered with scheme IV.

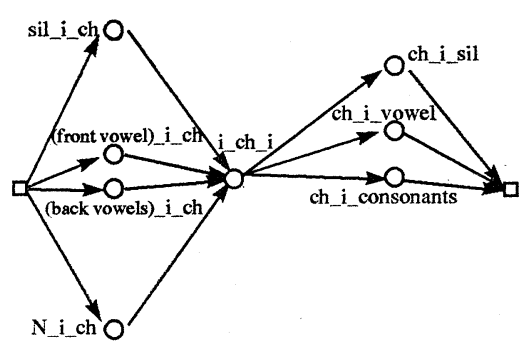

Fig. 10 Inter-word context-dependent word grammar for "ichi" (one in Japanese) clustered with scheme V.

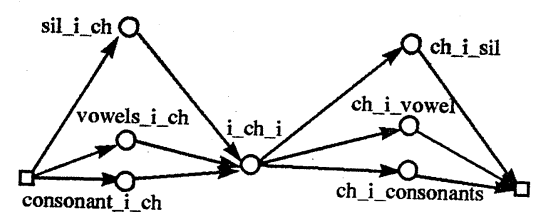

Fig. 11 Inter-word context-dependent word grammar for "ichi" (one in Japanese) clustered with scheme VI. schemes I through $\mathrm{V}$ and unclustered models were insignificant. Clustering schemes VI and VII performed significantly worse than others. These 
Table 3 Recognition error rates for inter-word context-dependent models with clustering (task-dependent).

\begin{tabular}{cccccccc}
\hline \multirow{2}{*}{$\begin{array}{c}\text { Clustering } \\
\text { scheme }\end{array}$} & $\begin{array}{c}\text { Number of } \\
\text { models }\end{array}$ & \multicolumn{3}{c}{ Error rate } & \multicolumn{2}{c}{$\begin{array}{c}\text { Score } \\
\text { buffers }\end{array}$} \\
\cline { 3 - 7 } & & Substitution & Deletion & Insertion & Word & Sentence & \\
\hline None & 461 & 1.0 & 0.4 & 0.3 & 1.7 & 13.3 & 1208 \\
I & 320 & 1.0 & 0.4 & 0.3 & 1.7 & 12.9 & 1012 \\
II & 370 & 1.0 & 0.4 & 0.3 & 1.8 & 13.3 & 1084 \\
III & 268 & 1.1 & 0.4 & 0.3 & 1.8 & 13.2 & 934 \\
IV & 222 & 1.2 & 0.3 & 0.4 & 1.9 & 14.0 & 800 \\
V & 222 & 1.1 & 0.3 & 0.4 & 1.8 & 13.6 & 799 \\
VI & 198 & 1.2 & 0.3 & 0.4 & 2.0 & 14.7 & 694 \\
VII & 146 & 1.2 & 0.4 & 0.5 & 2.0 & 15.1 & 534 \\
\hline
\end{tabular}

${ }_{1}$ Average number of score buffer slots needed per input speech frame.

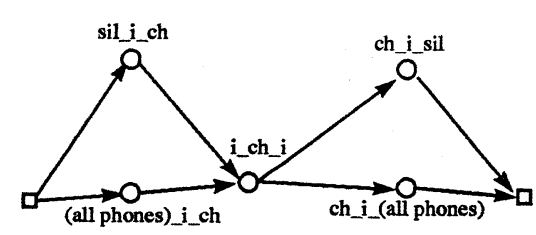

Fig. 12 Inter-word context-dependent word grammar for "ichi" (one in Japanese) clustered with scheme VII.

observations were tested and proven using two significance tests that are becoming popular, the matched pair test and the McNemar sign test. ${ }^{10,11)}$ Thus, at least with the current task, clustering scheme IV or $\mathrm{V}$ seems to be preferable since with these two schemes, it is possible to reduce the largest number of models without significant increase in the number of errors. The number of models is reduced from 461 for the unclustered case to 222 with these schemes, i.e. approximately $50 \%$ reduction. The number of average score buffer slots needed, in other words the average number of memory locations needed to store the path score and the previous grammar node per input frame of speech, decreases as the number of models decreases. Thus, less memory consumption, and as a consequence, less searches are expected with the proposed scheme.

\subsection{Evaluation of Inter-Word Context Clustering with Task-Independent Models}

We also tested the same inter-word contextdependent models with models trained on a different tasks than the ones tested on. As mentioned in Section 3, these models were trained on continuously read sentences. Unlike the task-dependent case, we cannot expect enough occurrences of inter-word context required in the test task to appear in the training task corpus. Thus, we need to use some measure to obtain enough occurrences of the required context. We tested two schemes. In both schemes, we assumed that the inter-word coarticulation is similar to word-internal coarticulation, and did not distinguish these two types of context. Therefore, we used word-internal context as well as inter-word context to obtain the required inter-word context-dependent models.

The first scheme pools all appropriate models with the required left or right context. The feature vectors of pooled models, consisting of feature elements such as LPC parameters, energy measures, etc. as has been explained in detail in Section 3.2, are then averaged after being weighed by their relative occurrence. In other words,

$$
V_{\text {pooled }}=\frac{\sum_{i}^{N} \sum_{j}^{M} C_{i j} V_{i j}}{\sum_{i, j}^{N, M} C_{i j}}
$$

Where, $V_{\text {pooled }}$ is the feature vector of the pooled model, $V_{i j}$ is the $j$-th feature vector in model $i$ assuming all models are numbered in sequence by $i$, and $C_{i j}$ is the relative occurrence of each vector, in other words the number of times the $j$-th vector in model $i$ was aligned with input speech during training. The transition probability is also updated from the weighted number of transitions found in the pooled models in a similar manner. Since throughout this paper we are using a single full covariance over all models, we assume that the 


\section{K. KONDO et al.: CLUSTERED INTER-PHRASE/WORD CONTEXT-DEPENDENT MODELS}

covariance matrix is not affected by the above operation. With this scheme, which we shall call the "pooling scheme," it is possible to obtain interword context-dependent models without retaining.

The second scheme involves pre-training of each inter-word context on both the left and the right side. Two additional training sessions on top of the conventional training for normal triphone models are run. One session is executed to obtain clustered inter-word context on the right side, while the other session is executed to obtain interword context on the left side. Each of these sessions include only the triphone models with clustered context on the desired side. In other words, the triphone models in one training session all have clustered context on the right only, and vice versa. All three session use the same training data. To give an example, assume that triphone models required for the word "ichi (one in Japanese)" are being trained. For this example, one training session will be run to obtain triphone models ${ }^{*}{ }_{-} i_{-} \mathrm{ch}_{\mathrm{i}}$, $i_{-}$ch_i $i_{i}$, and ch_i_* ${ }_{i}$, where $*$ designates don't care, and the subscript ${ }_{i}$ designates the training session to be for word-internal triphones. As will be explained later on, all models except $i_{-}$ch_ $i_{i}$ trained in this session will be discarded. Another session will be run to obtain all possible left inter-word context for the word "ichi," namely sil_i_ch, $\mathrm{N} \_$i_ch $_{1}$, (high vowel)_i_ch ${ }_{1}$, (mid-low vowel)_i_ch 1 , i_ch_i $i_{1}$, and ch_i_* ${ }_{1}$, where sil designates silence context. The last session will be run to obtain all possible right inter-word context, namely *_i_ch ${ }_{r}, i_{-} c_{-} i_{r}, c_{-} i_{-}$ vowel $_{r}$, ch_i_consonant ${ }_{r}$, and ch_i_sil $r$. After all training is finished, word models are constructed by extracting the required right and left inter-word context models from the appropriate model library, and the remaining word-internal context-dependent models from the original model library. Again, using the same example as above, the models for the word "ichi" will be composed from triphone models sil_i_ch $, \quad \mathrm{N}_{1} i_{-} \mathrm{ch}_{1}, \quad$ (high vowel)_i_ch, (mid-low vowel)_i_ch,$i_{1} \_c h \_i_{i}$, ch_i_vowel ${ }_{r}$, ch_i_consonant ${ }_{r}$, and ch_i_sil $l_{r}$. All other trained models will not be used here. We will call this scheme the "parallel training scheme."

Table 4 summarizes the results. We only tested inter-word context-dependent models without clustering and with clustering scheme V. Taskdependent models, i.e., models trained on digit strings were also included for comparison. Using these task-dependent models, we tested with conventional models mentioned in the previous section, and the "pool clustered" model mentioned in this section. Models with limited amount of training were also included to balance the amount of training with task-independent models.

Table 4 Recognition error rates for task-independent and dependent models.

\begin{tabular}{|c|c|c|c|c|c|c|c|c|}
\hline \multirow{2}{*}{$\begin{array}{c}\text { Task } \\
\text { dependency }\end{array}$} & \multirow{2}{*}{$\begin{array}{c}\text { Context } \\
\text { dependency }\end{array}$} & \multirow{2}{*}{$\begin{array}{l}\text { Context } \\
\text { clustering }\end{array}$} & \multirow{2}{*}{$\begin{array}{l}\text { Trained } \\
\text { frames }^{1}\end{array}$} & \multicolumn{5}{|c|}{ Error rate } \\
\hline & & & & Substitution & Deletion & Insertion & Word & Sentence \\
\hline \multirow{6}{*}{ Dependent } & Independent & 一 & $2,701,232$ & 1.8 & 0.4 & 0.4 & 2.6 & 18.7 \\
\hline & \multirow{5}{*}{ Dependent } & No & $2,623,359$ & 1.2 & 0.4 & 0.4 & 1.9 & 14.7 \\
\hline & & Case $V^{2}$ & $2,616,196$ & 1.3 & 0.3 & 0.4 & 2.1 & 15.3 \\
\hline & & Case $\mathrm{V}^{3}$ & $2,770,291$ & 1.5 & 0.4 & 0.4 & 2.3 & 17.1 \\
\hline & & No & 551,023 & 1.2 & 0.3 & 0.5 & 2.0 & 16.2 \\
\hline & & Case $\mathrm{V}^{3}$ & 559,914 & 1.3 & 0.4 & 0.4 & 2.1 & 16.5 \\
\hline \multirow{4}{*}{ Independent } & Independent & 一 & 443,812 & 5.3 & 2.1 & 2.1 & 9.5 & 48.5 \\
\hline & \multirow{3}{*}{ Dependent } & No & 525,157 & 2.8 & 1.2 & 1.4 & 5.4 & 33.3 \\
\hline & & Case $V^{3}$ & 532,959 & 3.1 & 1.4 & 1.5 & 6.0 & 37.0 \\
\hline & & Case $V^{4}$ & $600,504^{5}$ & 3.6 & 1.3 & 1.2 & 6.0 & 37.0 \\
\hline
\end{tabular}

1 Total number of frames used in training models for the test vocabulary. Substituted monophones not included.

2 Trained with clustered context.

3 Pool clustered.

4 Parallel training applied.

${ }^{5}$ Includes frames being used more than once due to three parallel training sessions. 
With the same inter-word context modeling methods, the error rate for the task-independent models are about three times greater than for taskdependent models. For example, the word error rate for the inter-word context-independent models is $9.5 \%$ and $2.6 \%$ for task-independent and taskdependent models respectively. This seems to hold even when the amount of training for the taskdependent models are limited to comparable amount with task-independent models (approximately 500,000 frames). In other words, we found that the amount of training did not change the performance of the task dependent models significantly. For example, the word error rate with no clustering was $1.9 \%$ and $2.0 \%$ for full-set training $(2,623,359$ trained frames) and limited training $(551,023$ trained frames) respectively. Therefore, the difference between the task-dependent and independent models is not caused by the difference in the amount of training. The tripling of word error rate for taskindependent models are consistent with those reported by Hon. ${ }^{12)}$ We do plan to investigate more about the cause of this significant error increase. However, the subject is out of the scope of the current paper.

The amount of word error reduction with interword context-dependent models without clustering, compared to inter-word context-independent models, is clearly more drastic for task-independent models, with $43 \%$ reduction for task-independent models (from $9.5 \%$ to $5.4 \%$ ), and $27 \%$ for task-dependent models (from $2.6 \%$ to $1.9 \%$ ). The reason for this probably is that modeling inter-word context in task-independent models mitigate the cause of the tripling of errors stated in the last paragraph, most probably the inadequacy of training data. However, by clustering the inter-word context, there is a significant increase in word errors for task-independent models $(5.4 \%$ to $6.0 \%)$. With task-dependent models, clustering does not significantly degrade performance (from $1.9 \%$ to $2.1 \%$ for full-set trained models, and from $2.0 \%$ to $2.1 \%$ for limited-set trained models). Even with these degradations due to clustering, the error reduction with inter-word context-dependent models is more drastic with task independent models, with $36.8 \%$ error reduction, than task dependent models, with $23 \%$ reduction.

We also tested the difference between pooling the inter-word context into clusters with the "pooling scheme," and training inter-word context models from scratch using task-dependent training. The word error rate for these two cases were $2.3 \%$ and $2.1 \%$ respectively. Thus, the difference in the performance of these models is insignificant. The two context-dependent model creation schemes with task-independent models, namely the "pooling scheme" and the "parallel training scheme" were also tested. The results showed exactly identical word error rates and sentence error rates of $6.0 \%$ and $37.0 \%$ respectively. The breakdown of the errors, in other words, the substitution, deletion and insertion errors making up the total errors were somewhat different.

\section{CONCLUSION}

In this work, inter-word context-dependent models were proposed and tested. The models were trained with a sentence grammar which supervises both the inter-word and word-internal phonetic context. The recognition grammar allows paths to all interword context-dependent triphones in parallel, and poses no constraints on the search path. Even with this simple grammar, it was possible to reduce the error rate by $32 \%$ compared to models which do not model the inter-word context for task-dependent models. We proposed clustering schemes for the inter-word context. It was found that by clustering all consonants into one class and vowels into two classes, the total number of models required can be halved, while the error rate increase was kept insignificant.

We also tested the same inter-word contextdependent models and its clustering methods on task-independent models. The decrease in the error rate was even more drastic at $43 \%$. However, with clustered models, the word error rate increases significantly compared to unclustered models. Two methods to create clustered inter-word context models were tested; the first creates clustered interword context-dependent models by averaging phonetic models to be clustered with weighting according to the relative frequency of each feature vector in each model. The second creates models by running multiple training sessions, one to create left inter-word context-dependent models only, and another to create right inter-word context-dependent models only. These two methods showed no significant difference in performance.

Although the tests conducted here were for Japanese, we believe that similar methods can be 


\section{K. KONDO et al.: CLUSTERED INTER-PHRASE/WORD CONTEXT-DEPENDENT MODELS}

applied to other languages as well. The clustering scheme as well as its efficiency will differ, however. We plan to undergo more experiments to find this out.

\section{ACKNOWLEDGMENTS}

The authors would like to thank Dr. Joseph Picone for his initial guidance in this work. They also thank Nozomi Arai for her validation of the digit corpus which proved crucial for our experiments. They also thank Dr. Donald P. Shaver, Dr. Don W. Shaw, and Dr. Toshiaki Ikoma for continuing to support our work. Finally, we also thank members of the Audio and Speech Processing group at Tsukuba Research and Development Center, as well as members of the Speech Research Branch in the Systems and Information Science Laboratory for their discussions and encouragements.

\section{REFERENCES}

1) M.-Y. Hwang, H.-W. Hon, and K.-F. Lee, "Modeling between-word coarticulation in continuous speech recognition," Proc. Eurospeech 89, Paris, France, 005-008 (1989).

2) D. B. Paul, "The Lincoln continuous speech recognition system: Recent development and results," Proc. DARPA Speech and Natural Language Process. Workshop, Philadelphia, PA, 160-166 (1989).

3) R. Cardin, Y. Normandin, and E. Millien, "Interword coarticulation modeling and MMIE training for improved connected digit recognition," Proc. ICASSP 93, II-243-II-246 (1993).

4) T. Watanabe, R. Isotani, and S. Tsukada, "Speakerindependent speech recognition based on Hidden Markov Model using demi-syllable units," IEICE Trans. Part D-II J75-D-II, 1281-1289 (1992) (in Japanese).

5) W. Chou, T. Matsuoka, B.-H. Juang, and C.-H. Lee, "An algorithm of high resolution and efficient multiple string hypothesization for continuous speech recognition using inter-word models," Proc. ICASSP 94, II-153-II-156 (1994).

6) K. Itou, S. Hayamizu, and H. Tanaka, "Continuous speech recognition by context-dependent phonetic HMM and an efficient algorithm for finding $\mathrm{N}$-best sentence hypothesis," Proc. ICASSP 92, I-21-I-24 (1992).

7) K. Kondo, J. Picone, and B. Wheatley, "Comparative analysis of Japanese and English digit recognition," Proc. ICASSP 94, I-101-I-104 (1994).

8) T. Kobayashi, S. Itahashi, S. Hayamizu, and T. Takezawa, "ASJ continuous speech corpus for research," J. Acoust. Soc. Jpn. (J) 48, 888-893 (1992) (in Japanese).
9) P. K. Rajasekaran and G. R. Doddington, "Speech recognition in the F-16 cockpit using principal speetral components," Proc. ICASSP 85, 882-885 (1985).

10) L. Gillick and S. J. Cox, "Some statistical issues in the comparison of speech recognition algorithms," Proc. ICASSP 89, 532-535 (1989).

11) S. Nakagawa and H. Takagi, "Some methods for comparing pattern recognition algorithms and comments on evaluating speech recognition performance," J. Acoust. Soc. Jpn. (J) 50, 849-854 (1994) (in Japanese).

12) H.-W. Hon, "Vocabulary-independent speech recognition: The VOCIND system," Tech. Rep. CMUCS-92-108, Comput. Sci. Dept. Carnegie Mellon Univ., Pittsburgh, PA (April 1992).

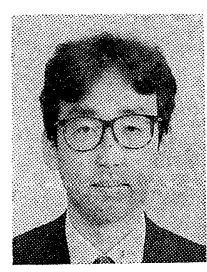

Kazuhiro Kondo received the B.E. and M.E. degrees in electrical engineering from Waseda University in 1982 and 1984 respectively. From 1984 to 1992 , he was with the Central Research Laboratory, Hitachi Ltd., Tokyo, Japan. During this time, he was engaged in research on speech coding and multiplexing schemes for communication, and video coding for digital VCRs. In 1992, he joined the Texas Instruments Tsukuba Research and Development Center Ltd., Ibaraki, Japan. From 1995, he has been on leave to the Systems and Information Science Laboratory, Texas Instruments Inc., Dallas, Texas. His current research interest include speech recognition and multimedia signal processing. Mr. Kondo is a member of the IEEE and the Institute of Electronics, Information, and Communication Engineers of Japan.

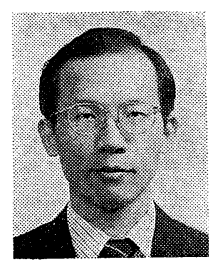

Yu-Hung Kao received the BS degree from the National Taiwan University in 1986, the MS and Ph.D. degrees from the University of Maryland, College Park, in 1990 and 1992 respectively, all in electrical engineering. From 1988 to 1992 , he received a Fellowship from Institute for Systems Research at University of Maryland. His research interests include speech compression and recognition. Thesis work on low complexity speech coding won an US patent and the university's Outstanding Invention Award of 1991. Since 1989, Dr. Kao has been with the speech research group at Texas Instruments. His work includes speech recognition, speech compression, speaker identification, and processing for environmental robustness. $\mathrm{He}$ is especially interested in the productization of the speech technology. For example, his work directly contributed to Sprint Voice FonCard (speech recognition and speaker verification), digital telephone answering machine (speech compression), and full duplex speaker phone (echo cancellation). 
Barbara Wheatley received a Ph.D. in Linguistics from Indiana University in 1981 and an M.S. in Computer Science from the University of Wisconsin-Milwaukee in 1988. She is currently a Senior Member of Technical Staff in Speech Research at Texas Instruments. She has worked on various aspects of speech recognition, including automatic enrollment, phonetic modeling, and word-spotting for telecommunications applications. Her recent work focuses on multilingual recognition systems, including cross-language adaptation and multilingual speech database development and analysis. 